\section{Diagnostic Criteria for Spontaneous Spinal CSF Leaks and Intracranial Hypotension}

We read with interest the paper by Schievink et al, ${ }^{1}$ in which the authors report a new set of diagnostic criteria for spontaneous spinal CSF leaks and intracranial hypotension encompassing its varied clinical and radiographic manifestations.

It has become well established that the clinical spectrum of spontaneous intracranial hypotension (SIH) is unusually varied. ${ }^{2}$ The wide range of clinical features and variations in patient response to treatment, as well as a lack of specific MR imaging findings, makes SIH difficult to diagnose. Previously, the only set of diagnostic criteria for spontaneous spinal CSF leak and intracranial hypotension was that published by the International Classification of Functioning, Disability and Health. ${ }^{3}$ Recently, it was appreciated that the International Headache Society (IHS) diagnostic criteria for SIH were based on a very small set of patients. We believe that the suggested new set of diagnostic criteria by Schievink et al is well designed. We would, however, like to comment on a few details with regard to these criteria.

Dr. Schievink and coauthors defined 3 main criteria as A, B, and C. Spinal meningeal diverticulum is considered the main criterion in B and C. This criterion describes whether there is a meningeal diverticulum but does not describe any details about the characteristics of a meningeal diverticulum. We believe that it may cause confusion because we have noticed many patients having small diverticulum without SIH, especially in the lower dorsal and upper lumbar regions. Considering this observation, we think that the criteria should include details about a meningeal diverticulum such as its location, morphologic features, size, and the number of diverticula.
A second characteristic, a response to an epidural blood patch, is also considered a main criterion in B and C. However, the authors did not mention response timing with regard to this. IHS sets these criteria as positive if a response to treatment is achieved in 72 hours. In our daily practice, we realized that the 72-hour criteria are not applicable in every patient. Therefore, we believe that the authors should define the time criterion for response to treatment to avoid diagnostic confusion such as 6 hours, 24 hours for temporary recovery, or full recovery.

In conclusion, we believe that the proposed set of diagnostic criteria will be useful in the diagnosis of a wide spectrum of SIH.

\section{References}

1. Schievink WI, Maya MM, Louy C, et al. Diagnostic criteria for spontaneous spinal CSF leaks and intracranial hypotension. AJNR Am J Neuroradiol. 2008; 29;853-56. Epub 2008 Feb 7

2. Mokri B, Posner JB. Spontaneous intracranial hypotension: the broadening clinical and imaging spectrum of CSF leaks. Neurology 2000;55:1771-72

3. Headache Classification Subcommittee of the International Headache Society. The International Classification of Headache Disorders: 2nd ed. Cephalalgia 2004;24 Suppl 1:9-160

S. Albayram

H. Ozer

B. Kara

Department of Radiology and Division of Neuroradiology Cerrahpasa Medical School

Istanbul, Turkey

DOI 10.3174/ajnr.A1156 